\title{
Editorial: Magnetic Flux Ropes: From the Sun to the Earth and Beyond
}

\author{
Rui Liu ${ }^{1,2,3 *}$, Jie Zhang ${ }^{4}$, Yuming Wang ${ }^{1,2}$ and Hongqiang Song ${ }^{5}$ \\ ${ }^{1}$ CAS Key Laboratory of Geospace Environment, Department of Geophysics and Planetary Sciences, University of Science and \\ Technology of China, Hefei, China, ${ }^{2}$ CAS Center for Excellence in Comparative Planetology, Hefei, China, ${ }^{3}$ Mengcheng National \\ Geophysical Observatory, School of Earth and Space Sciences, University of Science and Technology of China, Hefei, China, \\ ${ }^{4}$ Department of Physics and Astronomy, George Mason University, Fairfax, VA, United States, ${ }^{5}$ School of Space Science and \\ Physics, Shandong University, Weihai, China
}

Keywords: solar physics, interplanetary physics, space physics, solar magnetism, coronal mass ejection, interplanetary coronal mass ejection

\section{Editorial on the Research Topic:}

Magnetic Flux Ropes: From the Sun to the Earth and Beyond

OPEN ACCESS

Edited by:

Scott William McIntosh,

National Center for Atmospheric Research (UCAR), United States

Reviewed by:

Abhishek Kumar Srivastava, Indian Institute of Technology (BHU),

India

*Correspondence:

Rui Liu

rliu@ustc.edu.cn

Specialty section:

This article was submitted to Frontiers in Astronomy and Space Sciences, a section of the journal Frontiers in Stellar and Solar Physics

Received: 14 September 2020 Accepted: 09 October 2020

Published: 16 November 2020

Citation:

Liu R, Zhang J, Wang $Y$ and Song $H$ (2020) Editorial: Magnetic Flux Ropes:

From the Sun to the Earth and Beyond.

Front. Astron. Space Sci. 7:605957.

doi: 10.3389/fspas.2020.605957
The eruptions in the solar atmosphere exhibit distinctly diverse patterns across a vast range of spatio-temporal scales, from ever-expanding large-scale coronal mass ejections (CMEs), to localized flares within active regions harboring sunspots, to collimated jets down to the resolution limit of modern telescopes. As the core structure of various eruptive phenomena in the solar atmosphere, magnetic flux ropes, which are characterized by coherently twisted magnetic field lines, hold the key to understanding the physical mechanisms of solar eruptions and to our predictive capability of space weather. The purpose of this Frontiers Research Topic on magnetic flux ropes is to provide a forum to bring together multi-wavelength remote sensing and in-situ diagnostics, to integrate observation and numerical modeling, and to confront established models with new observations. The articles published in this Topic represent the most active fronts of research on a few important questions, namely, how flux ropes originate and evolve toward destabilization and beyond, how they are structured, and how they interact with each other and with surrounding magnetic fields and plasma. Below we briefly summarize the major results achieved by these articles.

Solar filaments (or prominences if appearing above the solar limb) are an important indicator of magnetic flux ropes on the Sun, although it has been controversially debated how a filament is magnetically structured, more specifically, whether the dense filament mass is supported against gravity by magnetic dips in a flux rope or a sheared arcade. Since the coronal magnetic field is extremely difficult to measure, filament mass serves as a valuable field tracer, especially when the individual threads are resolved and their dynamic motions are monitored. In equilibrium, dense filament plasmas may only trace a portion of magnetic field lines, but when disturbed, they must flow dominantly along field lines in a low- $\beta$ plasma environment. Mainly in chromospheric $\mathrm{H} \alpha$ filtergrams, signatures of flux ropes in the lower atmosphere have been detected by meter-class ground-based telescopes with a spatial resolution as high as 0.1 to 0.2 arcsec [see the review article by Wang and Liu, 2019]. Combining Doppler maps with flow maps in the plane of sky, [Awasthi and Liu, 2019] revealed a complex yet organized flow pattern inside a bubble underneath a prominence, which could be envisaged as counterstreaming mass motions in a helically distorted field resulting from the internal kink mode $m=2$.

Magnetic twist is an inherent property of flux ropes. A CME flux rope is often envisaged to build up like an onion with nested layers of magnetic flux added sequentially as the eruption progresses, which 
leads naturally to a non-uniform twist profile. Heating and dynamic motions as well as substructures are expected inside non-uniformly twisted flux ropes. MHD simulations of the prominence-cavity system [Fan and Liu, 2019] corroborated that there exist different types of twisted field lines threading the cavity. These field lines also possess different thermodynamic properties, therefore giving rise to the substructures of different appearances in EUV. In contrast to moderately twisted flux ropes (less than 2 turns) that are normally reconstructed within active regions by non-linear force-free field (NLFFF) extrapolations, [Jiang et al., 2019] found a highly twisted magnetic flux rope (over 6 turns) that connects two active regions, but a different NLFFF extrapolation fails to yield a flux rope in the same region, which poses again two controversial questions: 1 ) how the different NLFFF results can be reconciled? 2) what is the role of the kink instability in solar eruptions? To bear on the question as to how much twist a kinkstable flux rope could contain, [Liu et al., 2019] studied magnetic twist in rotational solar coronal jets and found a lower limit of 1.3 turns of twist as released by the rotation.

The evolution of magnetic flux ropes near the Sun and in interplanetary space has significant space-weather implications. [Vršnak, 2019] studied the pre-eruptive gradual rise of flux ropes with an analytical model, considering three different driving processes. [Ma and Chen, 2020] investigated two Type II radio bursts associated with three slow CMEs. [Mishra et al, 2020] made an effort to extrapolate the internal thermodynamic properties of a CME flux rope near the Sun to $1 \mathrm{AU}$ by using a model constrained by the CME kinematics. They concluded that the CME of interest releases heat throughout its journey from the Sun to Earth. Employing spacecrafts orbiting Venus and at the L1 point close to Earth, [Kilpua et al., 2019] found two CMEs coalesce into one coherent flux rope. [Zhao et al., 2019] concluded

\section{REFERENCES}

Awasthi, A. K., and Liu, R. (2019). Mass motion in a prominence bubble revealing a kinked flux rope configuration. Front. Phys. 7, 218. doi:10.3389/fphy.2019.00218.

Fan, Y., and Liu, T. (2019). Mhd simulation of prominence-cavity system. Front. Astron. Space Sci. 6, 27. doi:10.3389/fspas.2019.00027.

Jiang, C., Duan, A., Feng, X., Zou, P., and Wang, Y. (2019). Reconstruction of a highly twisted magnetic flux rope for an inter-active-region X-class solar flare. Front. Astron. Space Sci. 6, 63. doi:10.3389/fspas.2019.00063.

Kilpua, E. K. J., Good, S. W., Palmerio, E., Asvestari, E., Lumme, E., Ala-Lahti, M., et al. (2019). Multipoint observations of the june 2012 interacting interplanetary flux ropes. Front. Astron. Space Sci. 6, 50. doi:10.3389/fspas.2019.00050.

Liu, J., Wang, Y., and Erdélyi, R. (2019). How many twists do solar coronal jets release? Front. Astron. Space Sci. 6, 44. doi:10.3389/fspas.2019.00044.

$\mathrm{Ma}, \mathrm{S}$., and Chen, H. (2020). Two successive type ii radio bursts associated with b-class flares and slow cmes. Front. Astron. Space Sci. 7, 17. doi:10.3389/fspas.2020.00017.

Mishra, W., Wang, Y., Teriaca, L., Zhang, J., and Chi, Y. (2020). Probing the thermodynamic state of a coronal mass ejection (cme) up to 1 au. Front. Astron. Space Sci. 7, 1. doi:10.3389/fspas.2020.00001. that the coalescence process between interplanetary CME flux ropes can operate in scales of hundreds of Earth radii and persist for hundreds of minutes.

In this Research Topic, we also identify two important trends: 1) utilizing high-resolution observations [e.g., Awasthi and Liu, 2019; Wang and Liu, 2019], and 2) integrating observations with models [e.g., Fan and Liu, 2019; Jiang et al., 2019; Kilpua et al., 2019; Mishra et al., 2020]. To further advance our understanding of the origin, structure, and evolution of magnetic flux ropes in the heliosphere, we look forward to observations obtained by next-generation instruments such as the 4-m Daniel K. Inouye Solar Telescope, Parker Solar Probe (PSP), Solar Orbiter, as well as the Advanced Space-based Solar Observatory (ASO-S) that is scheduled to launch in 2022 by China.

\section{AUTHOR CONTRIBUTIONS}

RL drafted the editorial. All authors contributed to its finalization.

\section{FUNDING}

RL acknowledge support by the National Natural Science Foundation of China (NSFC; Grant Nos. 41761134088, 41774150, and 11925302).

\section{ACKNOWLEDGMENTS}

We acknowledge all the authors, reviewers, editors, and publishers who have supported this Research Topic.

Vršnak, B. (2019). Gradual pre-eruptive phase of solar coronal eruptions. Front. Astron. Space Sci. 6, 28. doi:10.3389/fspas.2019.00028.

Wang, H., and Liu, C. (2019). Signatures of magnetic fFront. Astron. Space Sci.lux ropes in the low solar atmosphere observed in high resolution. Front. Astron. Space Sci. 6, 18. doi:10.3389/fspas.2019.00018.

Zhao, Y., Feng, H., Liu, Q., and Zhao, G. (2019). Coalescence of magnetic flux ropes within interplanetary coronal mass ejections: multi-cases studies. Front. Phys. 7, 151. doi:10.3389/fphy.2019.00151.

Conflict of Interest: The authors declare that the research was conducted in the absence of any commercial or financial relationships that could be construed as a potential conflict of interest.

Copyright (c) 2020 Liu, Zhang, Wang and Song. This is an open-access article distributed under the terms of the Creative Commons Attribution License (CC BY). The use, distribution or reproduction in other forums is permitted, provided the original author(s) and the copyright owner(s) are credited and that the original publication in this journal is cited, in accordance with accepted academic practice. No use, distribution or reproduction is permitted which does not comply with these terms. 